Bull. Soc. math. France

131 (4), 2003, p. 587-601

\title{
DIFFERENTIAL GALOIS THEORY FOR AN EXPONENTIAL EXTENSION OF $\mathbb{C}((z))$
}

\author{
By Magali Bouffet
}

\begin{abstract}
In this paper we study the formal differential Galois group of linear differential equations with coefficients in an extension of $\mathbb{C}((z))$ by an exponential of integral. We use results of factorization of differential operators with coefficients in such a field to give explicit generators of the Galois group. We show that we have very similar results to the case of $\mathbb{C}((z))$.

RÉSumÉ (Théorie de Galois différentielle). - On étudie le groupe de Galois différentiel formel d'équations différentielles linéaires dont les coefficients sont dans une extension exponentielle de $\mathbb{C}((z))$. On utilise des résultats de factorisation d'opérateurs différentiels à coefficients dans un tel corps pour expliciter des générateurs du groupe de Galois. On obtient des résultats très similaires au cas du corps $\mathbb{C}((z))$.
\end{abstract}

\section{Introduction}

The motivation of this work is to write a local differential Galois theory for linear differential equations with coefficients admitting essential singularities. The aim is to generalize the case of differential equations having germs of meromorphic functions as coefficients. We only treat here the formal case, and work with the field $K=\mathbb{C}((z))$ of formal power series. It is a natural idea to add to this field exponential functions, as these ones are the new functions that appear to build solutions of equations with coefficients in $K$.

Texte reçu le 20 décembre 2002, accepté le 6 février 2003

Magali Bouffet, IWR, Im Neuenheimer Feld 368, D-69120 Heidelberg (Allemagne)

E-mail : bouffet@picard.ups-tlse.fr

2000 Mathematics Subject Classification. - 12H05, 13N10.

Key words and phrases. - Differential Galois theory, linear differential equations, exponential extension, universal differential extension, differential Galois group. 
Let's first fix some notations. We endow the field $K$ with the derivation

$$
\delta=-z^{2} \frac{\mathrm{d}}{\mathrm{d} z} \text {. }
$$

We set

$$
X=\mathrm{e}^{1 / z} \quad \text { and } \quad L=\mathbb{C}((z))((X)) .
$$

We extend the derivation $\delta$ to $L$ by $\delta(X)=X$. We also endow the field $K$ with the $z$-adic valuation $v_{z}$ and the field $L$ with the $X$-adic valuation $v_{X}$. We notice that this field is complete with respect to this valuation. We want to study linear differential equations with coefficients in $L$ from the differential Galois theory viewpoint. In particular we want to determine the structure of the differential Galois group. For this we proceed like for the field $K$ and we obtain very similar results. Let's recall the well-known results for the field $K$.

DeFinition 1.1. - We call a universal differential extension of a differential field $k$ a $k$-algebra $R$ satisfying the following conditions:

- the derivation defined on $k$ extends to $R$;

- $R$ is simple (i.e. $R$ has no non-trivial differential ideal);

- every homogeneous linear differential equation with coefficients in $k$ has all its solutions in $R$;

- $R$ is minimal, that is $R$ is generated over $k$ by all the solutions (and their derivatives) of homogeneous linear differential equations with coefficients in $k$.

For any differential field such an extension exists and is unique up to differential isomorphism. In the case of $K$, we can explicitly write the universal differential extension. It is given by the following symbols:

$$
R=\mathbb{C}((z))\left[\left\{z^{m}\right\}_{m \in \mathbb{C}},\{e(p)\}_{p \in \mathcal{P}}, \ell\right]
$$

where $\mathcal{P}=\bigcup_{n \geq 1} z^{-1 / n} \mathbb{C}\left[z^{-1 / n}\right]$, and the following relations:

$$
z^{a+b}=z^{a} z^{b}, \quad e\left(p_{1}+p_{2}\right)=e\left(p_{1}\right) e\left(p_{2}\right)
$$

and $z^{a}=z^{a} \in \mathbb{C}((z))$ for $a \in \mathbb{Z}$. The derivation on $R$ is given by

$$
\left(z^{a}\right)^{\prime}=a z^{a}, \quad(e(p))^{\prime}=p e(p), \quad \ell^{\prime}=1 .
$$

(We also write' the derivation $z \mathrm{~d} / \mathrm{d} z$ on $\mathbb{C}((z)))$.

We can interpret the preceding symbols as functions, which makes sense on suitable sectors. The symbol $z^{a}$ can be interpreted as the function $\mathrm{e}^{a \log (z)}, \ell$ as a logarithm function and $e(p)$ as the function $\exp \left(\int p / z \mathrm{~d} z\right)$.

Once we have the universal differential extension of $K$ we define $K$ differential automorphisms of $R$ as follows:

- the formal monodromy $\gamma$ is defined by

$$
\gamma\left(z^{a}\right)=\mathrm{e}^{2 i \pi a} z^{a}, \quad \gamma(\ell)=\ell+2 i \pi, \quad \gamma(e(p))=e(\gamma(p)) ;
$$

TOME $131-2003-\mathrm{N}^{\mathrm{O}} 4$ 
- the exponential torus is defined by: for all $h \in \operatorname{Hom}\left(\mathcal{P}, \mathbb{C}^{*}\right), \sigma_{h}$ is given by

$$
\sigma_{h}\left(z^{a}\right)=z^{a}, \quad \sigma_{h}(\ell)=\ell, \quad \sigma_{h}(e(p))=h(p) e(p) .
$$

Then the exponential torus and the formal monodromy generate the differential Galois group of the extension $R / \mathbb{C}((z))$ as a pro-algebraic group.

We show in this paper that we have the same kind of result for the field $L$. We give an explicit description of the universal differential extension of $L$ and we give topological generators of the differential Galois group as a pro-algebraic group.

The first thing to do is then to determine all the solutions of homogeneous linear differential equations with coefficients in $L$. For this we show that it suffices to solve order 1 homogeneous and non-homogeneous equations with coefficients in the algebraic closure of $L$. Let's write

$$
\widehat{K}_{\infty}=\bigcup_{n \geq 1} \mathbb{C}\left(\left(z^{1 / n}\right)\right) \text {. }
$$

Then $\widehat{K}_{\infty}$ is the algebraic closure of $K$. The algebraic closure of $L$ is

$$
\widehat{L}_{\infty}=\bigcup_{m \geq 1, n \geq 1} \mathbb{C}\left(\left(z^{1 / n}\right)\right)\left(\left(X^{1 / m}\right)\right) .
$$

(The previous valuations and derivations extend to these fields). We notice that the field of constants of $\widehat{L}_{\infty}$ as well as the one of all intermediate differential fields is $\mathbb{C}$.

We need the following result of factorization that can be found in [1]:

Theorem. - Let $P \in \widehat{L}_{\infty}[\delta]$ a non constant differential operator. Then $P$ can be factored in product of order 1 operators in the ring $\widehat{L}_{\infty}[\delta]$.

A right factor immediately gives a formal solution by solving an equation of the type $\delta(y)=a y$. We show by analyzing this factorization that either the factors "commute" in a certain sense, either some order 1 non-homogeneous equations appear.

\section{Formal classification of differential equations}

We want to determine the solutions of all linear differential equations with coefficients in $L$, but we are interested only in "new" solutions that are not already in $\widehat{L}_{\infty}$. Thus we start by classifying order 1 equations over $\widehat{L}_{\infty}$.

\subsection{Order 1 equations}

Homogeneous equations. - We want to classify equations of the type $\delta(y)=a y$, with $a \in \widehat{L}_{\infty}$. 
DEFINITION 2.1.1. - The equations $\delta(y)=$ ay and $\delta(y)=b y$, with $a, b \in \widehat{L}_{\infty}$, are said to be equivalent over $\widehat{L}_{\infty}$ if there exists $f \in \widehat{L}_{\infty} \backslash\{0\}$ such that $b-a=\delta(f) / f$. (The solutions of $\delta(y)=$ ay are then the solutions of $\delta(y)=b y$ multiplied by $f$.)

To classify the order 1 homogeneous equations we have to determine the set

$$
\mathcal{L} o g=\left\{\delta(f) / f, f \in \widehat{L}_{\infty}\right\}
$$

to study the quotient $\widehat{L}_{\infty} / \mathcal{L}$ og. Some computations show that

$$
\begin{aligned}
& \mathcal{L} o g=\left\{\lambda+\mu z+\sum_{r>0} \alpha_{r} z^{1+r / n}+\sum_{r>0} \beta_{r} X^{r / m}\right. \\
&\left.n, m \geq 1, \lambda, \mu \in \mathbb{Q}, \alpha_{r} \in \mathbb{C}, p \geq 1, \beta_{r} \in \mathbb{C}\left(\left(z^{1 / p}\right)\right)\right\} .
\end{aligned}
$$

Let $M$ be a $\mathbb{Q}$-vector space such that $M \oplus \mathbb{Q}=\mathbb{C}$. We set:

$$
\begin{aligned}
& \mathcal{Q}=\bigcup_{m \geq 1, n \geq 1} X^{-1 / m} \mathbb{C}\left(\left(z^{1 / n}\right)\right)\left[X^{-1 / m}\right], \\
& \mathcal{P}=\left\{\bigcup_{n \geq 1} z^{-1 / n} \mathbb{C}\left[z^{-1 / n}\right]\right\} \cup \bigcup_{n \geq 1}\left\{\sum_{r=1}^{n-1} \alpha_{r} z^{r / n}, \alpha_{r} \in \mathbb{C}\right\} .
\end{aligned}
$$

Then $M \oplus M z \oplus \mathcal{P} \oplus \mathcal{Q}$ classifies the order 1 homogeneous linear differential equations with coefficients in $\widehat{L}_{\infty}$. We study each of these sets to define symbolic solutions. We have the following symbols:

$$
\left\{X^{m}\right\}_{m \in M}, \quad\left\{z^{m}\right\}_{m \in M}, \quad\{e(p)\}_{p \in \mathcal{P}}, \quad\{g(q)\}_{q \in \mathcal{Q}} .
$$

The solutions of the equation

$$
\delta(y)=(m+\tilde{m} z+p+q) y
$$

are given by

$$
y=a X^{m} z^{\widetilde{m}} e(p) g(g),
$$

with $a \in \mathbb{C}$, and those of the equivalent equations by $f y$, with $f \in \widehat{L}_{\infty} \backslash\{0\}$.

We observe that these symbols are algebraically independent over $\widehat{L}_{\infty}$.

As for the symbols defined to solve equations over the field $K$ these symbols can be interpreted as functions, which makes sense on suitable sectors. The symbols $X^{m}$ and $z^{m}$ can be interpreted as the function $\mathrm{e}^{m \log (X)}$ and $\mathrm{e}^{m \log (z)}$, the symbols $e(p)$ as the functions $\exp \left(\int-p / z^{2} \mathrm{~d} z\right)$ and the symbols $g(q)$ as the functions $\exp \left(\int-q / z^{2} \mathrm{~d} z\right)$. The symbols $e(p)$ correspond to the symbols we recalled in the introduction for the field $K$. (We wrote them $e(p)$ with $p \in \bigcup_{n \geq 1} z^{-1 / n} \mathbb{C}\left[z^{-1 / n}\right]$ with the derivation $z \mathrm{~d} / \mathrm{d} z$. The second set comes from this change of derivation. We do not obtain the function $\mathrm{e}^{1 / z}$ as it is already in the base field.) The symbols $g(q)$ are exponentials of "second level". 
Non homogeneous equations. - We want to classify equations of the type $\delta(y)=a$, with $a \in \widehat{L}_{\infty}$.

Definition 2.1.2. - The equations $\delta(y)=a$ and $\delta(y)=b$, with $a, b \in \widehat{L}_{\infty}$, are said to be equivalent over $\widehat{L}_{\infty}$ if there exists $f \in \widehat{L}_{\infty}$ such that $b-a=\delta(f)$. (The solutions of $\delta(y)=a$ are then the solutions of $\delta(y)=b$ added to $f$.)

We have to determine the set $\widehat{L}_{\infty} / \mathcal{D} e r$, where

$$
\operatorname{Der}=\left\{\delta(f), f \in \widehat{L}_{\infty}\right\} .
$$

Some computations show that the order 1 non-homogeneous equations are classified by

$$
\widehat{L}_{\infty} / \operatorname{Der}=\{\alpha z, \alpha \in \mathbb{C}\} .
$$

Thus we only have one equation to study. Let's look at $\delta(y)=-z$. We set $\ell$ the symbol solution of this equation. Then the solutions of all equivalent equations are given by $\ell+g, g \in \widehat{L}_{\infty}$. The set of solutions of all the equations $\delta(y)=f$, with $f \in \widehat{L}_{\infty}$, is $\left\{\alpha \ell+g, \alpha \in \mathbb{C}, g \in \widehat{L}_{\infty}\right\}$.

The symbol $\ell$ can be interpreted as a logarithm function and we notice that it is algebraically independent over $\widehat{L}_{\infty}$ with the other symbols.

2.2. Differential operators and differential modules. - Let's write $\mathcal{D}=\widehat{L}_{\infty}[\delta]$. Let $A \in \operatorname{Hom}\left(\left(\widehat{L}_{\infty}\right)^{n},\left(\widehat{L}_{\infty}\right)^{n}\right)$. We define the differential module $\mathcal{M}_{A}$ associated to the system $\delta(Y)=A Y$ by the formulas

$$
\delta\left(e_{i}\right)=-\sum_{j} a_{i j} e_{j},
$$

where $\left(e_{1}, \ldots, e_{n}\right)$ is the standard basis of $\left(\widehat{L}_{\infty}\right)^{n}$. To a differential operator $P=\delta^{n}+a_{n-1} \delta^{n-1}+\cdots+a_{0}$ we associate a differential system $\delta(Y)=A Y$ with

$$
A=\left(\begin{array}{ccccc}
0 & 1 & 0 & \cdots & 0 \\
0 & 0 & 1 & \ddots & \vdots \\
\vdots & & \ddots & \ddots & 0 \\
0 & \cdots & \cdots & 0 & 1 \\
-a_{0} & -a_{1} & \cdots & \cdots & -a_{n-1}
\end{array}\right) .
$$

The module $\mathcal{M}_{A}$ is called the differential module associated to the operator $P$. The modules $\mathcal{M}_{A}$ and $(\mathcal{D} / \mathcal{D} P)^{*}$ are isomorphic, where $(\mathcal{D} / \mathcal{D} P)^{*}=$ $\operatorname{Hom}\left((\mathcal{D} / \mathcal{D} P), \widehat{L}_{\infty}\right)$ is the dual of $(\mathcal{D} / \mathcal{D} P)$.

The two differential systems $\delta(Y)=A_{1} Y$ and $\delta(Y)=A_{2} Y$ are said equivalent over $\widehat{L}_{\infty}$ if there exists $U \in \operatorname{Gl}\left(\left(\widehat{L}_{\infty}\right)^{n},\left(\widehat{L}_{\infty}\right)^{n}\right)$ such that

$$
A_{1}=U^{-1} \delta(U)+U^{-1} A_{2} U .
$$

BULlETIN DE LA SOCIÉTÉ MATHÉMATIQUE DE FRANCE 
This means equivalently that the associated differential modules are isomorphic. In the case of order 1 differential equations we obtain:

$$
\begin{aligned}
(\delta(y)+a y=0 & \stackrel{\text { equi }}{\sim} \delta(y)+b y=0) \Longleftrightarrow b-a \in \mathcal{L} \text { og } \\
& \Longleftrightarrow \mathcal{D} / \mathcal{D}(\delta+a) \stackrel{\text { iso }}{\sim} \mathcal{D} / \mathcal{D}(\delta+b) .
\end{aligned}
$$

DeFinition 2.2.1. - An operator $P \in \mathcal{D}$ is said reducible over $\widehat{L}_{\infty}$ if it can be written $P=P_{1} P_{2}$ with the orders of $P_{1}$ and $P_{2}$ strictly less to the order of $P$. It is said irreducible otherwise.

The theorem of factorization recalled in the introduction shows that the operators irreducible over $\mathcal{D}$ are those of order 1 .

Proposition 2.2.2. - The operator $P \in \mathcal{D}$ is reducible over $\widehat{L}_{\infty}$ if and only if the $\mathcal{D}$-module $\mathcal{D} / \mathcal{D} P$ contains a proper submodule. (The $\mathcal{D}$-module $\mathcal{D} / \mathcal{D} P$ is then said reducible, and irreducible otherwise.)

The irreducible $\mathcal{D}$-modules are those of dimension 1.

Definition 2.2.3. - A $\mathcal{D}$-module $\mathcal{M}$ is said decomposable if there exists two submodules $\mathcal{M}_{1}$ and $\mathcal{M}_{2}$ different from $\mathcal{M}$ and non-zero such that $\mathcal{M}=\mathcal{M}_{1} \oplus \mathcal{M}_{2}$. Otherwise $\mathcal{M}$ is said indecomposable. (An operator is said decomposable if the $\mathcal{D}$-module $\mathcal{D} / \mathcal{D} P$ is decomposable.)

In the next subsection we determine the indecomposable $\mathcal{D}$-modules (or operators).

2.3. Formal classification. - We recalled in the introduction that any non constant monic $P \in \mathcal{D}$ can be written

$$
P=\left(\delta+x_{1}\right) \cdots\left(\delta+x_{n}\right)
$$

with $x_{i} \in \widehat{L}_{\infty}$. Let's study now the uniqueness of this factorization.

Definition 2.3.1. - An element $a \in \widehat{L}_{\infty}$ is said non regular is it satisfies one of the following conditions:

- $v_{X}(a)<0$

- $v_{X}(a)=0$ and $\forall q \in \mathbb{Q}, v_{z}\left(a_{0}+q\right)<1$

- $v_{X}(a)=0, \exists q \in \mathbb{Q}, v_{z}\left(a_{0}+q\right)=1$ and $\forall r \in \mathbb{Q},\left(a_{0}+q\right)_{1}+r \neq 0$.

The following results are shown in [2]:

- If $a$ is non regular, then for any $b \in \widehat{L}_{\infty}$ the equation $\delta(u)+a u=b$ admits a unique solution $u$ in $\widehat{L}_{\infty}$.

- If $a$ is non regular, then the equation $u^{2}+\delta(u)+a u=-\delta(a)$ admits a solution $u$ in $\widehat{L}_{\infty}$ with $u$ regular. (If $\delta(a)$ is non zero then this solution is unique.)

TOME $131-2003-\mathrm{N}^{\mathrm{O}} 4$ 
These results are proved looking at the valuations of the different terms appearing in the equation.

We notice that the set of regular elements corresponds to the set $\mathcal{L}$ og defined in Section 2.1. In particular $a, b$ regular $\Rightarrow a+b$ regular.

Proposition 2.3.2. - Let $P \in \mathcal{D}$ of order $n \geq 1$. Let $\left(\delta+x_{1}\right) \cdots\left(\delta+x_{n}\right)$ and $\left(\delta+y_{1}\right) \cdots\left(\delta+y_{n}\right)$ be two factorizations of $P$ in the ring $\mathcal{D}$. Then there exists a permutation $\sigma$ of $\{1, \ldots, n\}$ such that $x_{i}-y_{\sigma(i)}$ is a regular element for every $i$.

Proof. - We saw in the previous subsection that the element $x_{i}-y_{\sigma(i)}$ is regular if and only if the two $\mathcal{D}$-modules $\mathcal{D} / \mathcal{D}\left(\delta+x_{i}\right)$ and $\mathcal{D} / \mathcal{D}\left(\delta+y_{\sigma_{i}}\right)$ are isomorphic. Let's consider the Jordan-Hölder sequence of $\mathcal{D} / \mathcal{D} P$ :

$$
\mathcal{D} / \mathcal{D} P \supset \mathcal{D}\left(\delta+x_{n}\right) / \mathcal{D} P \supset \cdots \supset \mathcal{D}\left(\delta+x_{2}\right) \cdots\left(\delta+x_{n}\right) / \mathcal{D} P \supset\{0\} .
$$

We set $\mathcal{M}_{0}=\mathcal{D} / \mathcal{D} P, \mathcal{M}_{1}=\mathcal{D}\left(\delta+x_{n}\right) / \mathcal{D} P, \ldots, \mathcal{M}_{n}=\{0\}$. Each quotient $\mathcal{M}_{i} / \mathcal{M}_{i+1}$ is isomorphic to $\mathcal{D} / \mathcal{D}\left(\delta+x_{n-i}\right)$ and each of these differential modules is simple. The Jordan-Hölder theorem tells that if we have another sequence up to a permutation the quotients are isomorphic. This gives the permutation $\sigma$ required.

Let's study now the decomposition of the $\mathcal{D}$-module $\mathcal{D} / \mathcal{D} P$.

First we notice that if $x_{i}-x_{j}$ is regular then the factors $\left(\delta+x_{i}\right)$ and $\left(\delta+x_{j}\right)$ "commute", that is they commute up to a regular element. We can write

$$
\left(\delta+x_{i}\right)\left(\delta+x_{j}\right)=\left(\delta+x_{j}+A\right)\left(\delta+x_{i}-A\right)
$$

with $A$ regular. To find $A$ one has to solve the following equation

$$
A^{2}+\delta(A)+\left(x_{j}-x_{i}\right) A=-\delta\left(x_{j}-x_{i}\right) .
$$

Since $A$ is regular the two factorizations are different. We can make the factors commute this way. Hence we put together the factors whose difference is a regular element, in making them commute with the factors with which they have a non regular element as difference. Thus we can write:

$$
P=\left(\delta+x_{1}\right) \cdots\left(\delta+x_{i_{1}}\right) \cdots\left(\delta+x_{i_{p}+1}\right) \cdots\left(\delta+x_{i_{p+1}}\right),
$$

with the following properties: for all $j, 0 \leq j \leq p$, we write $I_{j}$ the integer interval $\left[x_{i_{j}+1}, \ldots, x_{i_{j+1}}\right]$, then if $k, \ell$ are in the same interval $I_{j}$ the element $x_{k}-x_{\ell}$ is regular, and if $k, \ell$ are in two different intervals $I_{j}$ the element $x_{k}-x_{\ell}$ is non regular. The different blocks corresponding to the intervals $I_{j}$ commute and this leads to the following decomposition:

$\mathcal{D} / \mathcal{D} P \stackrel{\text { iso }}{\sim} \mathcal{D} / \mathcal{D}\left(\delta+x_{1}\right) \cdots\left(\delta+x_{i_{1}}\right) \oplus \cdots \oplus \mathcal{D} / \mathcal{D}\left(\delta+x_{i_{p}+1}\right) \cdots\left(\delta+x_{i_{p+1}}\right)$.

We now have to study the case where $x_{i}-x_{j}$ is a regular element.

BULlETIN DE LA SOCIÉTÉ MATHÉMATIQUE DE FRANCE 
REMARK 2.3.3. - Even if we don't have yet the complete description of the $\mathcal{D}$-module $\mathcal{D} / \mathcal{D} P$ we can draw some conclusions about the solutions of the differential equation $P(y)=0$. The preceding decomposition tells us that to solve the equation $P(y)=0$ one has to solve equations of the type

$$
\delta(y)+x_{i} y=0 \text { and }\left(\delta+x_{\ell}\right) \cdots\left(\delta+x_{k}\right)(y)=0,
$$

where in this case $x_{j}-x_{i}$ is a regular element for all $i, j$. (This gives the solutions modulo the equivalence relation defined in Section 2.2.) Since $x_{j}-x_{i}$ is regular the equations $\delta(y)+x_{i} y=0$ and $\delta(y)+x_{j} y=0$ are equivalent over $\widehat{L}_{\infty}$ and their solutions differ by multiplication by an element of $\widehat{L}_{\infty}$. Then we can solve by the constant variation method and we have to solve equations of the type $\delta(y)=x_{i} y$ and $\delta^{r}(\lambda)=f$ with $f \in \widehat{L}_{\infty}$. From the classification of non homogeneous order 1 equations we know that if $f \in \mathcal{D}$ er the equation $\delta(\lambda)=f$ admits a solution in $\widehat{L}_{\infty}$, otherwise we obtain the symbol $\ell$. When iterating the operation we obtain as solutions polynomials in $\ell$ with coefficients in $\widehat{L}_{\infty}$ of degree at most $r$. Thus the solutions of the equation $P(y)=0$ are in the ring $\widehat{L}_{\infty}\left[\ell,\{\text { solutions of } \delta(y)+a y=0\}_{a \in \widehat{L}_{\infty}}\right]$. If $P$ is of order $n$ the polynomials in $\ell$ are of degree at most $n-1$.

Definition 2.3.4. - Let $a \in \widehat{L}_{\infty}$ be a regular element. There exists $f \in \widehat{L}_{\infty}$ such that $a=\delta(f) / f$. The element $a$ is said regular non rigid if $f \in \mathcal{D} e r$. It is said regular rigid otherwise.

The element $f$ is unique up to multiplication by a constant which does not change the fact of being in $\mathcal{D} e r$ or not.

EXAMPLE 2.3.5. - It is difficult to give an explicit description of the regular rigid elements but the following elements

$$
a=-z+\operatorname{hot}(z)+\operatorname{hot}(X) \text { and } a=-1-\alpha z X+\operatorname{hot}(X)
$$

with $\alpha \in \mathbb{C} \backslash\{0\}$ are regular rigid.

Proposition 2.3.6. - Let $a \in \widehat{L}_{\infty}$ regular. Then we have:

$a$ is non rigid $\Longleftrightarrow$ the equation $\delta(y)+a y=1$ admits a solution in $\widehat{L}_{\infty}$.

Proof. - Let $a$ regular non rigid. Since $a$ is regular the equation $\delta(y)+a y=0$ admits a solution $y_{a}$ in $\widehat{L}_{\infty}$. We set $y=\lambda y_{a}$ and we solve $\delta(y)+a y=1$ that is $\delta(\lambda)=y_{a}^{-1}$. We have $-a=\delta\left(y_{a}\right) / y_{a}$ hence $a=\delta\left(y_{a}^{-1}\right) / y_{a}^{-1}$. Since $a$ is non rigid $y_{a}^{-1} \in \mathcal{D} e r$ and the equation $\delta(\lambda)=y_{a}^{-1}$ admits a solution in $\hat{L}_{\infty}$.

Conversely assume that $\delta(y)+a y=1$ admits a solution $y_{b}$ in $\widehat{L}_{\infty}$. Since $a$ is regular the equation $\delta(y)+a y=0$ admits a solution $y_{a}$ in $\widehat{L}_{\infty}$ and $y_{b}-y_{a}$ is also a solution of the equation $\delta(y)+a y=1$. We write $y_{b}-y_{a}=\left(y_{c}-1\right) y_{a}$ with $y_{c}=y_{b} y_{a}^{-1} \in \widehat{L}_{\infty}$. Then $\delta\left(y_{c}\right)=y_{a}^{-1}$ hence $y_{a}^{-1} \in \operatorname{Der}$ and $a$ is non rigid.

TOME $131-2003-\mathrm{N}^{\mathrm{O}} 4$ 
Proposition 2.3.7. - Let $P=\left(\delta+x_{1}\right)\left(\delta+x_{2}\right) \in \mathcal{D}$ with $x_{2}-x_{1}$ regular, then:

$$
\begin{aligned}
x_{2}-x_{1} \text { is non rigid } & \Longleftrightarrow \mathcal{D} / \mathcal{D} P \stackrel{\text { iso }}{\sim} \mathcal{D} / \mathcal{D}\left(\delta+x_{1}\right) \oplus \mathcal{D} / \mathcal{D}\left(\delta+x_{2}\right), \\
x_{2}-x_{1} \text { is rigid } & \Longleftrightarrow \mathcal{D} / \mathcal{D} P \text { is indecomposable } \\
& \Longleftrightarrow \mathcal{D} / \mathcal{D} P \stackrel{\text { iso }}{\sim} \mathcal{D} / \mathcal{D}\left(\delta+x_{2}+z\right)\left(\delta+x_{2}\right) .
\end{aligned}
$$

Proof. - Let's consider the following exact sequence:

$$
0 \rightarrow \mathcal{D} / \mathcal{D}\left(\delta+x_{1}\right) \stackrel{\cdot\left(\delta+x_{2}\right)}{\longrightarrow} \mathcal{D} / \mathcal{D}\left(\delta+x_{1}\right)\left(\delta+x_{2}\right) \stackrel{\lambda}{\longrightarrow} \mathcal{D} / \mathcal{D}\left(\delta+x_{2}\right) \rightarrow 0,
$$

where $\cdot\left(\delta+x_{2}\right)$ is the product by $\left(\delta+x_{2}\right)$ to the right and $\lambda$ is the quotient application. This exact sequence splits if and only if we can solve the equation $1+\left(\delta+x_{2}\right) B=C\left(\delta+x_{1}\right)$ in $\mathcal{D}$, that is if we can solve the equation $\delta(y)+\left(x_{2}-x_{1}\right) y=1$ in $\widehat{L}_{\infty}$. Then from the preceding proposition we deduce that if $x_{2}-x_{1}$ is non rigid we have $\mathcal{D} / \mathcal{D} P \stackrel{\text { iso }}{\sim} \mathcal{D} / \mathcal{D}\left(\delta+x_{1}\right) \oplus \mathcal{D} / \mathcal{D}\left(\delta+x_{2}\right)$. On the contrary if $x_{2}-x_{1}$ is rigid the exact sequence does not split and the $\mathcal{D}$-module $\mathcal{D} / \mathcal{D} P$ is indecomposable.

If $x_{2}-x_{1}$ is regular rigid we have $x_{2}-x_{1}=\delta(f) / f$ with $f \in \widehat{L}_{\infty}$ and $f \notin \mathcal{D e r}$. Let $y_{2}$ be a solution of $\delta(y)+x_{2} y=0$. Then a basis of solutions of the equation $P(y)=0$ is given by $\left(y_{2}, \lambda y_{2}\right)$ with $\delta(\lambda)=f$. Since $f \notin \mathcal{D e r}$ we can write $\lambda=\alpha \ell+g$ with $\alpha \in \mathbb{C} \backslash\{0\}, g \in \widehat{L}_{\infty}$ and $\ell$ is the symbol defined in Section 2.1. Then a basis of solutions of $P(y)=0$ can be written $\left(y_{2},(\alpha \ell+g) y_{2}\right)$. Let $Q=\left(\delta+x_{2}+z\right)\left(\delta+x_{2}\right)$. A basis of solutions of the equation $Q(y)=0$ is given by $\left(y_{2}, \ell y_{2}\right)$. We can link these two basis by a change of basis with coefficients in $\widehat{L}_{\infty}$ hence these two equations are equivalent over $\widehat{L}_{\infty}$ and the corresponding $\mathcal{D}$-modules are isomorphic.

Conclusion. - The indecomposable $\mathcal{D}$-modules of dimension 2 are isomorphic to $\mathcal{D} / \mathcal{D}(\delta+x+z)(\delta+x)$.

Proposition 2.3.8. - Let $P=\left(\delta+x_{1}\right)\left(\delta+x_{2}\right) \cdots\left(\delta+x_{n}\right) \in \mathcal{D}$. Then:

$\mathcal{D} / \mathcal{D} P$ indecomposable $\Longleftrightarrow \mathcal{D} / \mathcal{D} P \stackrel{\text { iso }}{\sim} \mathcal{D} / \mathcal{D}(\delta+x+n z) \cdots(\delta+x+z)(\delta+x)$.

Proof. - We only consider the case where all the $x_{j}-x_{i}$ are regular since we have already seen that otherwise we can decompose the $\mathcal{D}$-module $\mathcal{D} / \mathcal{D} P$.

We can compute the solutions of the equation $P(y)=0$ as seen in Remark 2.3.3. The solutions of the equations $\delta(y)+x_{i} y=0$ are all equal up to multiplication by an element of $\widehat{L}_{\infty}$ as the $x_{j}-x_{i}$ are regular. Let $y_{0}$ be a solution of one of these equations. The solutions of the equation $P(y)=0$ are polynomials in $\ell$ of degree at most $n-1$ where $n$ is the order of the operator $P$. The coefficients of these polynomials are of the type $y_{0} f$ with $f \in \widehat{L}_{\infty}$. We can write a basis of solutions of $P(y)=0$ as

$$
\mathcal{B}_{P}=\left(y_{0} f_{0,0}, y_{0}\left(f_{1,0}+f_{1,1} \ell\right), \ldots, y_{0}\left(f_{n-1,0}+f_{n-1,1} \ell+\cdots+f_{n-1, n-1} \ell^{n-1}\right)\right)
$$


with $f_{i, j}$ in $\widehat{L}_{\infty}$. The operator $P$ is indecomposable if and only if $f_{n-1, n-1} \neq 0$. (In this case for all $i, f_{i, i} \neq 0$.)

Indeed, if $P$ is decomposable we can determine the solutions of $P(y)=0$ by solving equations of lower degree and we do not obtain terms in $\ell^{n-1}$. Conversely if there is no term in $\ell^{n-1}$ there exists an integer $m<n-1$ such that several polynomials of degree $m$ appear. Let's write

$$
\left(\tilde{y}_{0}, \ldots, \tilde{y}_{n-1}\right)=\left(y_{0} f_{0,0}, \ldots, y_{0}\left(f_{n-1,0}+\cdots+f_{n-1, n-1} \ell^{n-1}\right)\right)
$$

and assume that $\tilde{y}_{m}$ and $\tilde{y}_{m+1}$ are polynomials in $\ell$ with the same degree. Then there exists a change of basis with coefficients in $\widehat{L}_{\infty}$ that transforms $\mathcal{B}_{P}$ into $\left(\tilde{y}_{0}, \ldots, \tilde{y}_{m}, f y_{0}, \ldots, \tilde{y}_{n-1}\right)$ with $f$ and $f_{0,0}$ linearly independent over $\mathbb{C}$. Then the operator $P$ admits several right factors and is decomposable.

The operator $Q=(\delta+x+n z) \cdots(\delta+x+z)(\delta+x)$ is indecomposable since a basis of solutions is given by $\mathcal{B}_{Q}=\left(y_{0}, \lambda_{1} y_{0}, \ldots, \lambda_{n-1} y_{0}\right)$ with $\delta^{i}\left(\lambda_{i}\right)=z$ and $y_{0}$ a solution of the equation $\delta(y)+x y=0$. Any indecomposable operator $P$ is equivalent to $Q$ : since for all $i, f_{i, i} \neq 0$ a change a basis with coefficients in $\widehat{L}_{\infty}$ transforms $\mathcal{B}_{Q}$ into $\mathcal{B}_{P}$.

Conclusion. - From Krull-Schmidt theorem we have a unique decomposition in indecomposable $\mathcal{D}$-modules. Hence for all $P \in \mathcal{D}$ we have:

$\mathcal{D} / \mathcal{D} P \stackrel{\text { iso }}{\sim} \mathcal{D} / \mathcal{D}\left(\delta+x_{1}+n_{1} z\right) \cdots\left(\delta+x_{1}\right) \oplus \cdots \oplus \mathcal{D} / \mathcal{D}\left(\delta+x_{r}+n_{r} z\right) \cdots\left(\delta+x_{r}\right)$, with the $n_{i}$ in $\mathbb{N}$.

\section{Normal forms and differential systems}

We consider a differential system $\delta(Y)=A Y$ with $A \in \mathcal{M}_{n}(\widehat{L})$. Any linear differential equation can be written as a system (with a compagnon matrix), and conversely the cyclic vector lemma enables us to find a compagnon matrix (thus associated to an equation) in the conjugacy class of any system.

The preceding results for $\mathcal{D}$-modules can be translated to systems. Let $A \in \mathcal{M}_{n}(\widehat{L})$. There exists a matrix $T$ in $\mathrm{Gl}_{n}\left(\widehat{L}_{\infty}\right)$ such that the matrix

$$
A^{T}=T^{-1} A T-T^{-1} \delta(T)
$$

is diagonal block as follows:

$$
A=\left(\begin{array}{cccc}
B_{1} & 0 & \cdots & 0 \\
0 & B_{2} & \ddots & \vdots \\
\vdots & \ddots & \ddots & 0 \\
0 & \cdots & 0 & B_{r}
\end{array}\right), \quad \text { with } B_{i}=\left(\begin{array}{ccccc}
b_{i} & z & 0 & \cdots & 0 \\
0 & b_{i} & z & \ddots & \vdots \\
\vdots & \ddots & \ddots & \ddots & 0 \\
\vdots & \ddots & \ddots & b_{i} & z \\
0 & \cdots & \cdots & 0 & b_{i}
\end{array}\right),
$$

or $B_{i}=\left(b_{i}\right)$ with $b_{i} \in \widehat{L}_{\infty}$. 
We can assume that $b_{i} \in \mathcal{Q} \oplus \mathcal{P} \oplus M \oplus M z$. We can write $b_{i}=q_{i}+p_{i}+m_{i}+\tilde{m}_{i} z$ with the corresponding notations. We set:

$$
L_{i}=\left(\begin{array}{cccc}
m_{i} & 0 & \cdots & 0 \\
0 & \ddots & \ddots & \vdots \\
\vdots & \ddots & \ddots & 0 \\
0 & \cdots & 0 & m_{i}
\end{array}\right) \text { and } \tilde{L}_{i}=\left(\begin{array}{ccccc}
\tilde{m}_{i} & 1 & 0 & \cdots & 0 \\
0 & \ddots & \ddots & \ddots & \vdots \\
\vdots & \ddots & \ddots & \ddots & 0 \\
\vdots & \ddots & \ddots & \ddots & 1 \\
0 & \cdots & \cdots & 0 & \tilde{m}_{i}
\end{array}\right)
$$

Then there exists a matrix $Q_{i}$ with coefficients in $\mathcal{Q} \oplus \mathcal{P}$ such that a normal form of the system $\delta(Y)=B_{i} Y$ can be written $N_{i}=\exp \left(Q_{i}\right) X^{L_{i}} z^{\tilde{L_{i}}}$ where $X^{L_{i}}$ stands for $\exp \left(L_{i} \log (X)\right)$ and $z^{\tilde{L_{i}}}$ stands for $\exp \left(\tilde{L_{i}} \log (z)\right)$. A normal form of the system $\delta(Y)=A^{T} Y$ can then be written

$$
N=\left(\begin{array}{cccc}
N_{1} & 0 & \cdots & 0 \\
0 & N_{2} & \ddots & \vdots \\
\vdots & \ddots & \ddots & 0 \\
0 & \cdots & 0 & N_{r}
\end{array}\right)=\exp (Q) X^{L} z^{\tilde{L}}
$$

with obvious notations and a normal form of the system $\delta(Y)=A Y$ is given by $T \cdot N$.

\section{Formal differential Galois group}

4.1. Universal ring. - We can now build the universal differential extension of $\widehat{L}$. We consider the commutative algebra generated over $\widehat{L}_{\infty}$ by the previous symbols:

$$
R_{0}=\widehat{L}_{\infty}\left[\left\{X^{m}, z^{m}\right\}_{m \in M},\{e(p)\}_{p \in \mathcal{P}},\{g(q)\}_{q \in \mathcal{Q}}, \ell\right] .
$$

The ring $\widehat{L}_{\infty}$ is endowed with the derivation $\delta=-z^{2} \mathrm{~d} / \mathrm{d} z$. This derivation extends to $R_{0}$ by

$$
\begin{aligned}
& \delta\left(z^{m}\right)=-m z^{m+1}, \quad \delta\left(X^{m}\right)=m X^{m}, \quad \delta(e(p))=p e(p), \\
& \delta(g(q))=q g(q), \quad \delta(\ell)=-z .
\end{aligned}
$$

The ring $R_{0}$ is a differential ring. Let's consider the following ideal:

$$
\begin{gathered}
I=\left\langle X^{0}-1, z^{0}-1,\right. \\
\left\{X^{m_{1}+m_{2}}-X^{m_{1}} X^{m_{2}}, z^{m_{1}+m_{2}}-z^{m_{1}} z^{m_{2}}\right\}_{m_{1}, m_{2} \in \mathbb{C}}, \\
e(0)-1, g(0)-1,\left\{e\left(p_{1}+p_{2}\right)-e\left(p_{1}\right) e\left(p_{2}\right)\right\}_{p_{1}, p_{2} \in \mathcal{P}}, \\
\left.\left\{g\left(q_{1}+q_{2}\right)-g\left(q_{1}\right) g\left(q_{2}\right)\right\}_{q_{1}, q_{2} \in \mathcal{Q}}\right\rangle .
\end{gathered}
$$

Lemma 4.1.1. - The ideal $I$ is a differential ideal of $R_{0}$.

BULlETIN DE LA SOCIÉTÉ MATHÉMATIQUE DE FRANCE 
Then we consider the quotient $R=R_{0} / I$. We recall some well-known results (see [3]) we will use in the following proofs.

Lemma 4.1.2. - Let $(R, \delta)$ be a differential ring containing $\mathbb{Q}$. If $R$ is simple then it is an integral domain and its fraction field $L$ has the same ring of constants as $R$. (This ring is in fact a field.)

Lemma 4.1.3. - Let $(R, \delta)$ be a differential field with an algebraically closed field of constants and $(R, \delta)$ a differential ring over $K$. Assume that $R$ is simple and finitely generated over $K$. Then its fraction field $L$ has the same field of constants as $K$.

Lemma 4.1.4. - The ring $R=R_{0} / I$ is a simple differential ring. It is an integral domain and the field of constants of its fraction field is $\mathbb{C}$.

Proof. - We have to show that $R$ is simple, that is has no non-trivial differential ideal. Let's consider a particular subring of $R$. Let $m_{1}, \ldots, m_{s} \in M$, $\widetilde{m}_{1}, \ldots, \widetilde{m}_{\ell} \in M, q_{1}, \ldots, q_{t} \in \mathcal{Q}, p_{1}, \ldots, p_{r} \in \mathcal{P}$ linearly independent over $\mathbb{Q}$. We consider the subring $\widetilde{R}$ of $R$ generated by the $X^{m_{i}}, X^{-m_{i}}, z^{\widetilde{m}_{i}}, z^{-\widetilde{m}_{i}}$, $e\left(p_{i}\right), e\left(-p_{i}\right)$ and $g\left(q_{i}\right), g\left(-q_{i}\right)$. We start by proving that $\widetilde{R}$ is a simple differential ring. Let $J$ a differential ideal of $\widetilde{R}$ and assume that $J \neq 0$.

We call a monomial any element of the type $z^{\widetilde{m}} X^{m} e(p) g(q)$ with $m \in \mathbb{Z} m_{1}+$ $\cdots+\mathbb{Z} m_{s}, \widetilde{m} \in \mathbb{Z} \widetilde{m}_{1}+\cdots+\mathbb{Z} \widetilde{m}_{\ell}, p \in \mathbb{Z} p_{1}+\cdots+\mathbb{Z} p_{r}, q \in \mathbb{Z} q_{1}+\cdots+\mathbb{Z} q_{t}$. We write $\mathcal{A}$ the set of all monomials.

Then any element of $\widetilde{R}$ can be written $\sum_{a \in \mathcal{A} \text {,finite }} h_{a} a$ with $h_{a} \in \widehat{L}_{\infty}$. We notice that if $a=z^{\widetilde{m}} X^{m} e(p) g(q)$ then $\delta(a)=(-\widetilde{m} z+m+p+q) a=\alpha(a) a$ with $\alpha(a) \in \widehat{L}_{\infty}$. Assume that $J$ contains an element with only one monomial and let $h$ be such an element. Then we can write $h=h_{1} z^{\widetilde{m}} X^{m} e(p) g(q)$ and

$$
\left(h_{1}\right)^{-1} z^{-\widetilde{m}} X^{-m} e(-p) g(-q) \cdot h=1 \in J .
$$

Hence $J=\widetilde{R}$. Assume now that all elements of $J$ except 0 have at least two monomials and let $N$ be the minimal number of monomials that can have a non zero element of $J$. Let $h \in J$ with exactly $N$ monomials and write $h=\sum_{i=1}^{N} h_{i} a_{i}$ with $N>1, h_{i} \in \widehat{L}_{\infty}, a_{i} \in \mathcal{A}$. Up to a multiplication by an element of $\widetilde{R}$ we can assume that $h_{1}=1$ and $a_{1}=1$. Then

$$
\delta(h)=\sum_{i=2}^{N}\left(\delta\left(h_{i}\right)+h_{i} \alpha\left(a_{i}\right)\right) a_{i} \in J .
$$

This element has strictly less monomials than $h$ then we have $\delta(h)=0$ by minimality. Then it comes $\left(\delta\left(h_{N}\right)+h_{N} \alpha\left(a_{N}\right)\right) a_{N}=0$ and since $a_{N} \neq 0$ we have $\delta\left(h_{N}\right)+h_{N} \alpha\left(a_{N}\right)=0$ with $h_{N} \in \widehat{L}_{\infty}$. This is a contradiction. 
Let's consider now $\tilde{S}$ the subring of $R$ generated by $\widetilde{R}$ and $\ell$. Any element of $\tilde{S}$ can be written $h=\sum_{i=0}^{n} r_{i} \ell^{i}$ with $r_{i} \in \widetilde{R}$. Then

$$
\delta(h)=\sum_{i=0}^{n-1}\left(\delta\left(h_{i}\right)-(i+1) z h_{i+1}\right) \ell^{i}-\delta\left(h_{n}\right) \ell^{n} .
$$

Let's show that $\tilde{S}$ is a simple differential ring. Let $J$ be a differential ideal of $\tilde{S}, J \neq 0$. If $J$ contains an element of degree 0 in $\ell$ then $J=\tilde{S}$. Hence we assume that all the non zero elements of $J$ have a strictly positive degree in $\ell$ and let $N$ be the smallest possible degree. We consider all the elements of $J$ with degree $N$ and we write $J_{0}$ the set of the coefficients of $\ell^{N}$ of these elements. Then $J_{0}$ is a non-zero differential ideal of $\widetilde{R}$ hence $J_{0}=\widetilde{R}$. Then $J$ contains an element of the type $\sum_{i=0}^{N} r_{i} \ell^{i}$ with $r_{i} \in \widetilde{R}$ and $r_{N}=1$. Then

$$
\delta(h)=\sum_{i=0}^{N-1}\left(\delta\left(r_{i}\right)-(i+1) z r_{i+1}\right) \ell^{i} \in J . .
$$

By minimality of the degree we have $\delta(h)=0$ and in particular $\delta\left(r_{N-1}\right)-$ $N z r_{N-1}=0$, hence $\delta\left(r_{N-1}\right)=N z$ with $r_{N-1} \in \tilde{R}$ which is a contradiction. Hence $\tilde{S}$ is simple. The ring $R$ is the union of all the rings $\tilde{S}$ hence it is simple. Lemma 4.1.2 and 4.1.3 enable us to conclude.

Conclusion. - The ring $R$ is the universal differential extension of the field $\mathbb{C}((z))\left(\left(e^{1 / z}\right)\right)$.

4.2. Differential Galois group. - Let's write $F$ the fraction field of $R$. We are interested in the differential $\widehat{L}$-automorphisms of $F$. Let's consider the following automorphisms:

- Let $h \in \operatorname{Hom}\left(\mathcal{Q}, \mathbb{C}^{*}\right)$ a group homomorphism from $(\mathcal{Q},+)$ to $\left(\mathbb{C}^{*},.\right)$. We define $\sigma_{h}$ by

$$
\sigma_{h}(g(q))=h(q) g(q), \quad \forall q \in \mathcal{Q},
$$

and $\sigma_{h}$ leaves $\widehat{L}_{\infty}$ and the other generators of $R$ invariant. For $h \in \operatorname{Hom}\left(\mathcal{Q}, \mathbb{C}^{*}\right)$ the $\sigma_{h}$ form a group that we call the over exponential torus.

- Let $h \in \operatorname{Hom}\left(\mathcal{P}, \mathbb{C}^{*}\right)$ a group homomorphism from $(\mathcal{P},+)$ to $\left(\mathbb{C}^{*},.\right)$. We define $\tau_{h}$ by

$$
\tau_{h}(e(p))=h(p) e(p), \quad \forall p \in \mathcal{P},
$$

and $\tau_{h}$ leaves $\widehat{L}_{\infty}$ and the other generators of $R$ invariant. For $h \in \operatorname{Hom}\left(\mathcal{P}, \mathbb{C}^{*}\right)$, the $\tau_{h}$ form a group that we call the exponential torus.

- We set $\hat{\gamma}$ the formal monodromy in $z$ automorphism defined by

$$
\begin{array}{lll}
\hat{\gamma}\left(z^{m}\right)=\mathrm{e}^{2 i \pi m} z^{m}, & \forall m \in \mathbb{C} ; & \hat{\gamma}(e(p))=e(\hat{\gamma}(p)), \quad \forall p \in \mathcal{P} ; \\
\hat{\gamma}(g(q))=g(\hat{\gamma}(q)), \quad \forall q \in \mathcal{Q} ; & \hat{\gamma}(\ell)=\ell+2 i \pi
\end{array}
$$

and $\hat{\gamma}$ leaves the $X^{m}$ invariant. 
- We set $\hat{\Gamma}$ the formal monodromy in $X$ automorphism defined by

$$
\hat{\Gamma}\left(X^{m}\right)=e^{2 i \pi m} X^{m}, \forall m \in \mathbb{C} ; \quad \hat{\Gamma}(g(q))=g(\hat{\Gamma}(q)), \forall q \in \mathcal{Q}
$$

and $\hat{\Gamma}$ leaves $\ell$, the $z^{m}$ and the other generators of $R$ invariant.

The only relations between these automorphisms are the following:

- for all $h \in \operatorname{Hom}\left(\mathcal{P}, \mathbb{C}^{*}\right), \tau_{h} \circ \hat{\gamma}=\hat{\gamma} \circ \tau_{g}$ where $g=h \circ \hat{\gamma}$;

- for all $h \in \operatorname{Hom}\left(\mathcal{Q}, \mathbb{C}^{*}\right), \sigma_{h} \circ \hat{\gamma}=\hat{\gamma} \circ \sigma_{g}$ where $g=h \circ \hat{\gamma}$;

- for all $h \in \operatorname{Hom}\left(\mathcal{Q}, \mathbb{C}^{*}\right), \sigma_{h} \circ \hat{\Gamma}=\hat{\Gamma} \circ \sigma_{g}$ where $g=h \circ \hat{\Gamma}$.

Proposition 4.2.1. - If $f$ is an element of $F$ fixed by all the preceding differential automorphisms then $f \in \widehat{L}$.

Proof. - Let $f \in F$. We can write $f=f_{1} / f_{2}$ with $f_{1}, f_{2} \in R, f_{1} \wedge f_{2}=1$. There exists $m_{1}, \ldots, m_{s} \in M, \widetilde{m}_{1}, \ldots, \widetilde{m}_{\ell} \in M, q_{1}, \ldots, q_{t} \in \mathcal{Q}, p_{1}, \ldots, p_{r} \in \mathcal{P}$ linearly independent over $\mathbb{Q}$ such that $f_{1}$ and $f_{2}$ are in the subring of $R$ generated by $z^{\widetilde{m}_{i}}, z^{-\widetilde{m}_{i}}, X^{m_{i}}, X^{-m_{i}}, e\left(p_{i}\right), e\left(-p_{i}\right), g\left(q_{i}\right), g(-q(i))$ and $\ell$. We still call a monomial an element of the type

$$
z^{\widetilde{m}} X^{m} e(p) g(q)
$$

where $m \in \mathbb{Z} m_{1}+\cdots+\mathbb{Z} m_{s}, \tilde{m} \in \mathbb{Z} \widetilde{m}_{1}+\cdots+\mathbb{Z} \widetilde{m}_{s}, p \in \mathbb{Z} p_{1}+\cdots+\mathbb{Z} p_{r}$ and $q \in \mathbb{Z} q_{1}+\cdots+\mathbb{Z} q_{r}$.

We write $\mathcal{A}$ the set of all monomials and $f_{1}=\sum_{i=0}^{n_{1}} \alpha_{i} \ell^{i}, f_{2}=\sum_{i=0}^{n_{2}} \beta_{i} \ell^{i}$ with $\alpha_{i}=\sum_{j=1}^{N_{i}} \lambda_{i j} a_{i j}$ and $\beta_{i}=\sum_{j=1}^{M_{i}} \mu_{i j} b_{i j}$, where $\lambda_{i j}, \mu_{i j} \in \widehat{L}_{\infty}$ and $a_{i j}, b_{i j} \in \mathcal{A}$. We assume that the $N_{i}$ and $M_{i}$ are minimal and that $f$ is fixed by all the preceding differential automorphisms.

- Let $\sigma$ be an automorphism of the type $\sigma_{h}$ with $h \in \operatorname{Hom}\left(\mathcal{Q}, \mathbb{C}^{*}\right)$. We write $a_{i j}=z^{\widetilde{m}_{i j}} X^{m_{i j}} e\left(p_{i j}\right) g\left(q_{i j}\right)$ and $b_{i j}=z^{\widetilde{m}_{i j}^{\prime}} X^{m_{i j}^{\prime}} e\left(p_{i j}^{\prime}\right) g\left(q_{i j}^{\prime}\right)$. Then:

$$
\begin{gathered}
\sigma\left(f_{1}\right)=\sum_{i=0}^{n_{1}}\left(\sum_{j=1}^{N_{i}} h\left(q_{i j}\right) \lambda_{i j} z^{\widetilde{m}_{i j}} X^{m_{i j}} e\left(p_{i j}\right) g\left(q_{i j}\right)\right) \ell^{i}, \\
\sigma\left(f_{2}\right)=\sum_{i=0}^{n_{2}}\left(\sum_{j=1}^{M_{i}} h\left(q_{i j}^{\prime}\right) \mu_{i j} z^{\widetilde{m}_{i j}^{\prime}} X^{m_{i j}^{\prime}} e\left(p_{i j}^{\prime}\right) g\left(q_{i j}^{\prime}\right)\right) \ell^{i} .
\end{gathered}
$$

The hypothesis $\sigma(f)=f$ can be written

$$
\frac{\sigma\left(f_{1}\right)}{\sigma\left(f_{2}\right)}=\frac{f_{1}}{f_{2}}
$$

which leads to $h\left(q_{i j}\right)=h\left(q_{k \ell}^{\prime}\right)$ for all $i, j, k, \ell$, that is $\sigma\left(f_{1}\right)=c(h) f_{1}$ and $\sigma\left(f_{2}\right)=c(h) f_{2}$ with $c(h) \in \mathbb{C}$. In particular for $f_{1}$ we have $h\left(q_{i j}\right)=c(h)$ for all $i, j$ and all $h \in \operatorname{Hom}\left(\mathcal{Q}, \mathbb{C}^{*}\right)$. Hence the elements $q_{i j}$ are all identical and we have $f_{1}=g(q) \tilde{f}_{1}$ with $\tilde{f}_{1} \in R$ containing no term of the type $g(q)$. We 
also have $f_{2}=g(q) \tilde{f}_{2}$. Then the hypothesis $f_{1} \wedge f_{2}=1$ implies $g(q)=1$ that is $q=0$. Hence $f_{1}$ and $f_{2}$ contain no term of the type $g(q)$.

- Considering an automorphism of the type $\tau_{h}$ the same method leads to the fact that $f_{1}$ and $f_{2}$ contain no term of the type $e(p)$.

- Considering the automorphisms $\hat{\gamma}$ and $\hat{\Gamma}$ we can show that $f_{1}$ and $f_{2}$ contain no ramified term in $z$ and in $X$, as well as no $\ell$. Hence $f \in \widehat{L}$.

Conclusion. - The elements $\sigma_{h}, \tau_{h}, \hat{\gamma}, \hat{\Gamma}$ are all differential $\widehat{L}$-automorphisms of $F$, hence are elements of the Galois group of the extension $F / \widehat{L}$. Thanks to Proposition 4.2.1 and to the Galois correspondence we conclude that the Galois group of the extension $F / \widehat{L}$ is topologically generated by $\sigma_{h}, \tau_{h}, \hat{\gamma}, \hat{\Gamma}$ as a pro-algebraic group.

REMARK 4.3. - All that has been done here with an extension of $\mathbb{C}((z))$ by the exponential $\mathrm{e}^{1 / z}$ could have been done with any other exponential $\mathrm{e}^{\mu}$ with $\mu \in \mathbb{C}((z))$.

Acknowledgments. - I am grateful to Bernard Malgrange for numerous improvements in all this text.

\section{BIBLIOGRAPHY}

[1] Bouffet (M.) - Un lemme de Hensel pour les opérateurs différentiels, C. R. Acad. Sci. Paris Sér. I Math., t. 331 (2000), no. 4, pp. 277-280.

[2] _ Factorisation d'opérateurs différentiels à coefficients dans une extension liouvillienne d'un corps valué, Ann. Inst. Fourier, t. 52 (2002), no. 3, pp. 709-734.

[3] Magid (A.R.) - Lectures on Differential Galois Theory, University Lecture Series, vol. 7, American Math. Society, 1994.

BULlETIN DE LA SOCIÉTÉ MATHÉMATIQUE DE FRANCE 